\title{
ANÁLISIS TAFONÓMICO DE LOS RESTOS ÓSEOS DE OTÁRIDOS: EL CASO DE LA COSTA SUR DE TIERRA DEL FUEGO
}

\section{TAPHONOMIC ANALYSIS OF OTARID BONE REMAINS: THE CASE OF THE SOUTH COAST OF TIERRA DEL FUEGO}

\author{
María Paz Martinoli¹, María A. Gutiérrez², Atilio Francisco J. Zangrando ${ }^{3}$ \\ ${ }^{1}$ CADIC-CONICET. Laboratorio de Antropología. Bernardo Houssay 200 (9410), Ushuaia, \\ Argentina. E-mail: mpmartinoli@yahoo.com.ar \\ ${ }^{2}$ Instituto de Investigaciones Arqueológicas y Paleontológicas del Cuaternario Pampeano \\ (INCUAPA-CONICET), Facultad de Ciencias Sociales, Universidad Nacional del Centro de la \\ Provincia de Buenos Aires, Avda. del Valle 5737, B7400JWI Olavarría, Buenos Aires, Argentina. \\ E-mail: mgutierr@soc.unicen.edu.ar \\ ${ }^{3}$ CADIC-CONICET. Laboratorio de Antropología. Bernardo Houssay 200 (9410), Ushuaia, \\ Argentina. E-mail: fzangrando@cadic-conicet.gob.ar
}

Presentado: 09/11/2020

Aceptado: 04/05/2021

\section{Resumen}

Los análisis tafonómicos específicos sobre la frecuencia de partes anatómicas en conjuntos óseos de pinnípedos han sido menos frecuentes en comparación con los de mamíferos terrestres. Sin embargo, los estudios zooarqueológicos permiten señalar interdependencia entre los valores de densidad mineral ósea (DMO) y los indices de utilidad económica (\%MUI), lo cual conlleva la incertidumbre de ponderar las estrategias de transporte por sobre procesos postdepositacionales. La propuesta del presente trabajo es analizar de manera separada las trayectorias de preservación de los restos óseos según su potencial de supervivencia en conjuntos arqueológicos de pinnípedos de la región del canal Beagle. Las variables para determinar dicho umbral fueron definidas a través de información actualística de sectores costeros de Tierra del Fuego-Patagonia, la cual considera la preservación diferencial de elementos según sus valores de DMO y las preferencias de los carnívoros. Los resultados obtenidos indican que los procesos postdepositacionales jugaron un rol central en la formación de la mayor parte de los conjuntos analizados. Al considerar la incidencia de factores tafonómicos, las estrategias de transporte de pinnípedos no son fácilmente reconocidas a partir de los perfiles anatómicos y advierte sobre el alcance de los modelos de utilidad económica para efectuar interpretaciones arqueológicas.

Palabras clave: tafonomía, pinnípedos, potencial de supervivencia, canal Beagle. 


\begin{abstract}
Specific taphonomic analyses on the frequency of anatomical parts in pinniped bone assemblages have been less frequent compared to those of terrestrial mammals. However, zooarchaeological studies also indicate an interdependence between bone mineral density values (BMD) and economic utility indices (\%MUI), which advises the misconception of weighing transport strategies over postdepositional processes. In this article, we propose to analyse separately the preservation conditions of four pinniped archaeological assemblages from Beagle Channel according to skeletal element survival. Considering previous taphonomic studies from coastal sectors of Tierra del Fuego-Patagonia, two groups of elements are defined according to bone mineral density and pattern of carnivore consumption. Results indicate that postdepositational processes played a central role in most of the studied assemblages. Our conclusion is that transport strategies in pinnipeds are difficult to recognize due to the influence of taphonomic factors on anatomical profiles. In turn, this result should be considered for the use of economic utility models to perform archaeological interpretations.
\end{abstract}

Key words: taphonomy, pinnipeds, survival potential, Beagle Channel.

\title{
Introducción
}

La preservación de los restos óseos en contextos arqueológicos es el resultado de la tensión entre procesos naturales y la actividad humana. Además, las características estructurales propias de los huesos sobre los que actúan directamente distintos agentes son claves a la hora de analizar el registro arqueofaunístico (Behrensmeyer 1975; Lyman 1994). Tal es el caso de los procesos atricionales mediados por la densidad mineral ósea, así como la acción de diferentes agentes como los carnívoros (Belardi et al. 2012; Gutiérrez et al. 2010; Lyman 1994; Marean y Cleghorn 2003; Stiner 2002). Estos últimos producen fracturas y otras modificaciones, además del transporte / sustracción / acumulación de especímenes, que afectan las abundancias de elementos anatómicos (Binford 1981; Blumenschine 1988; Borrero 2004; Carlson y Pickering 2004; Estévez y Mameli 2000; Gutiérrez et al. 2016; Kaufmann 2016; Marean et al. 1992; Martin 1998; Mondini 2018; Stiner 1991).

En las últimas dos décadas, esta problemática ha sido gravitatoria en la interpretación de conjuntos zooarqueológicos de la Argentina. Especialmente en arqueología de costas de Pampa y Patagonia, esto es visible a partir de estudios tafonómicos sistemáticos centrados en restos de distintos vertebrados que incluyen mamíferos terrestres (especialmente de ungulados) (Alunni et al. 2017; Borrero 2007; Estévez y Mameli 2000; Rindel y Belardi 2006), cetáceos (Borella 2004), aves (Cruz 2007, 2015; Cruz et al. 2019) y peces (Frontini et al. 2019). Con respecto a los restos de otáridos, presas de cierta importancia económica en tal espacio durante el Holoceno y Antropoceno (Borella et al. 2017; Orquera y Piana 1999; Schiavini 1993; Zangrando et al. 2014), también han sido analizados desde un punto de vista tafonómico (Borella y Borrero 2010, 2019; Borella y Muñoz 2006; Borrero 2004; Cruz y Muñoz 2010; Muñoz 1996, 2003, 2008, 2009, 2011, 2014; Muñoz y Cruz 2014; Muñoz et al. 2013; Vázquez et al. 2019), aunque en menor medida en comparación con mamíferos terrestres. 
Cuando se comenzaron a estudiar de manera sistemática los restos óseos de pinnípedos en conjuntos arqueológicos, se propuso que no existe equifinalidad entre factores culturales y naturales en la formación de los conjuntos óseos de estos mamíferos marinos. Esta afirmación se basaba en dos factores principales (Lyman et al. 1992): a) los huesos presentan en promedio una mayor densidad mineral ósea en comparación con la de mamíferos terrestres de similares dimensiones; y b) su tendencia a presentarse completos en los depósitos arqueológicos en gran medida por la carencia de canal medular en los huesos largos, lo que no promovía su procesamiento para consumo humano. No obstante, el cúmulo de información que se ha generado en las últimas décadas para la identificación y el estudio de los restos óseos de pinnípedos a partir de marcos de referencia particulares (Borella et al. 2007, 2013, 2018; Lyman et al. 1992; Martinoli 2019; San Román 2009; Savelle et al. 1996; Stora 2002), así como observaciones efectuadas en función del estudio de conjuntos de pinnípedos provenientes de distintos contextos, indican que los perfiles anatómicos identificados pueden estar mediados por la densidad mineral ósea y por la acción de carnívoros (Borella et al. 2017; Borrero 2004; Martinoli 2017, 2018; Moreno 2008; Muñoz 1996, 2005, 2009; Nagaoka et al. 2008; Orquera y Piana 1999; Vázquez et al. 2019).

Por su parte, la información etnográfica y etnoarqueológica referida al tema sugiere que las carcasas de pinnípedos pequeños y medianos se transportaban completas a las áreas residenciales; incluso luego de sufrir un trozamiento inicial en el espacio de captura para facilitar su acarreo (Lyman et al. 1992; Rasmussen 1931; Whitridge 1990). Esto aplica también para cazadores-recolectores-pescadores que contaban con medios de navegación, mediante los cuales era posible disminuir los costos de transporte (Ames 2002). Aún si consideramos estas estrategias de transporte, es factible que una proporción significativa de la variabilidad observada en las frecuencias de partes anatómicas no sea reflejo de estas actividades (Lyman et al. 1992), sino de procesos que incluyen el decaimiento de los huesos mediados por la densidad ósea y la acción de agentes como los carnívoros (Marean y Cleghorn 2003).

El objetivo del presente trabajo es analizar desde un enfoque tafonómico restos óseos de otáridos provenientes de cuatro conjuntos arqueológicos generados a partir de excavaciones amplias en la costa sur de Tierra del Fuego y que han mostrado abundancias anatómicas variables, con una tendencia a la mayor presencia de porciones de la presa de bajo valor económico (Martinoli y Vázquez 2017; Martinoli 2018, 2019). De acuerdo a la propuesta de algunos autores (Lupo 2006; Marean y Cleghorn 2003), la pregunta que consideramos pertinente realizar es en qué medida los patrones previamente observados en los perfiles anatómicos se relacionan con la preservación diferencial de porciones con más probabilidades de sobrevivir a procesos destructivos naturales y/o con pautas de transporte selectivo. 


\section{Casos de estudio de la costa sur de Tierra del Fuego}

Hemos seleccionado cuatro conjuntos arqueológicos de pinnípedos provenientes de dos sectores de la costa sur de Tierra del Fuego (Figura 1), los cuales cubren distintos momentos de la secuencia de la región (Orquera y Piana 2020). Los restos que serán analizados en este trabajo corresponden a machos / hembras adultos y subadultos de la especie Arctocephalus australis; los pesos de estas presas varían entre 50 y 100 kg (Schiavini 1990; Vaz Ferreira y Vallejo 1981). No han sido considerados los huesos catalogados como juveniles o cachorros, ya que por la falta de osificación de muchas de las porciones que los constituyen, los tamaños y valores de densidad mineral ósea varían con respecto a los de individuos adultos (Borella et al. 2007, 2013).
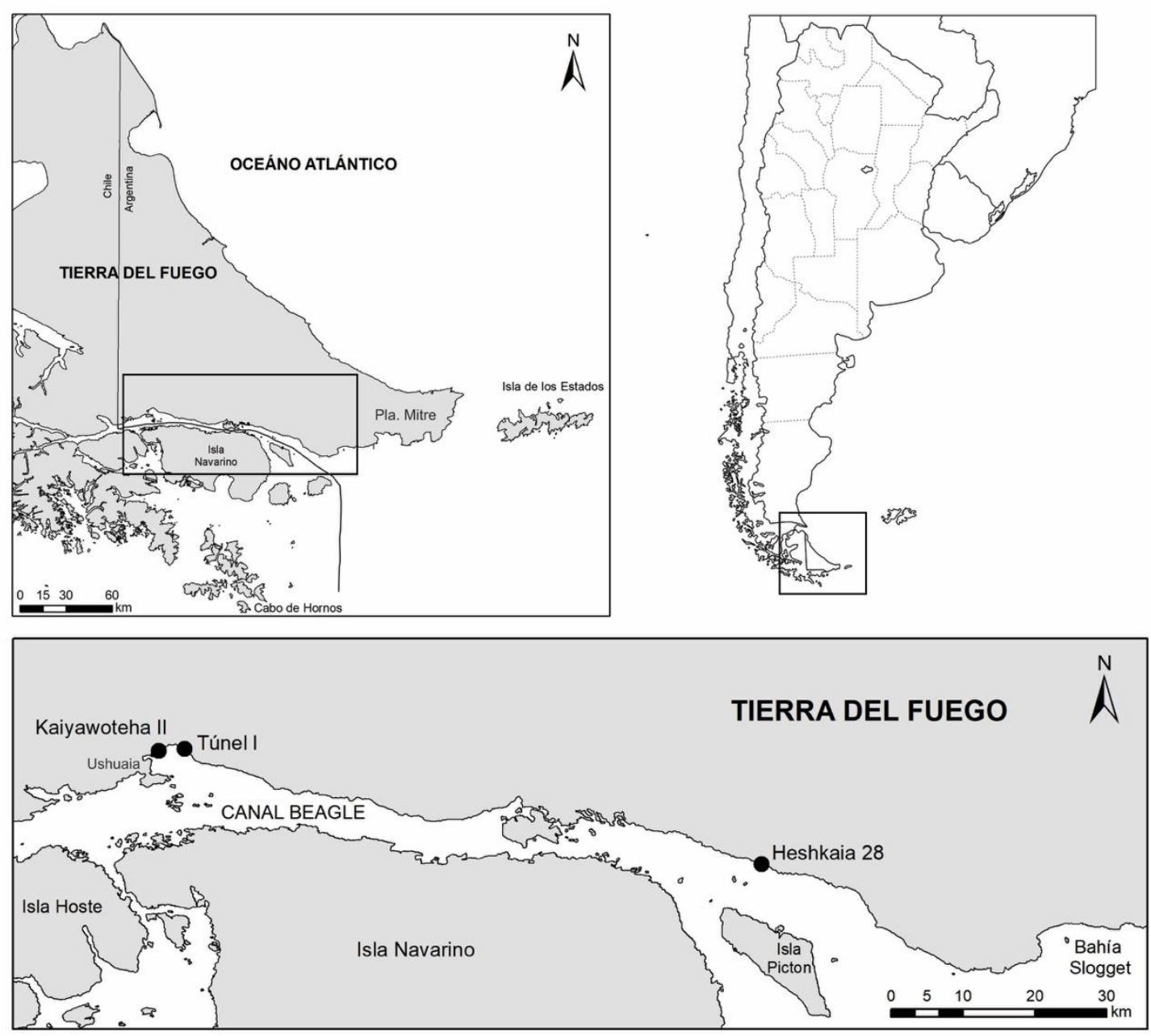

Figura 1. Mapa de Tierra del Fuego con indicación de los yacimientos analizados.

En la porción central del canal Beagle fueron elegidos tres conjuntos, los cuales

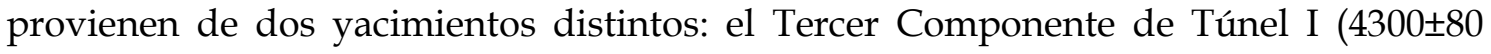
años AP, Orquera y Piana 1999) con una cronología del Holoceno medio y el Cuarto Componente de Túnel I (2660 100 años AP; Orquera y Piana 1999) y el sitio Kaiyawoteha II (730 \pm 45 años AP; Piana et al. 2007) que corresponden al Holoceno 
tardío. Las excavaciones de estos sitios abarcaron superficies amplias y la mayoría son concheros de distintas dimensiones. Estos tres conjuntos se caracterizan por la presencia general elevada de instrumentos óseos orientados hacia la explotación de recursos marinos (Orquera y Piana 1988; Piana et al. 2007). En lo que respecta al registro faunístico, los pinnípedos representan el mayor aporte energético a la dieta en los tres conjuntos, aunque presentan variabilidad en sus abundancias (Martinoli 2018; Piana et al. 2007; Schiavini 1990).

En la zona Moat estudiamos el conjunto proveniente del sitio Heshkaia 28. Este yacimiento es un conchero fechado en $678 \pm 38$ años AP (Zangrando et al. 2010), en el que se excavó una superficie de $9 \mathrm{~m}^{2}$. A diferencia de lo señalado para los conjuntos anteriores, en la localidad Heshkaia se registra un bajo aprovechamiento de pinnípedos. Esto condice con una baja frecuencia de instrumental especializado en la captura de estos recursos (Zangrando 2010). En cambio, se observa un mayor consumo de guanacos (Alunni et al. 2020; Alunni y Zangrando 2012) y una explotación de recursos litorales menores (aves, peces y moluscos) que es variable entre los conjuntos (Zangrando et al. 2014). El consumo de peces correspondientes a la familia Notothenidae se destaca particularmente en Heshkaia 28 (Corbat et al. 2020).

Respecto de las modalidades de explotación de las carcasas de pinnípedos, como se puede observar en la Tabla 1, uno de los aspectos que resalta es la diferencia en las abundancias anatómicas relevadas en cada uno de los conjuntos estudiados. Tales variaciones fueron interpretadas previamente como consecuencia de la selección de distintas estrategias de transporte y procesamiento en función de la estructura de los recursos (Martinoli 2018). Éstas implicarían el transporte diferencial de porciones en el caso de la zona Moat, considerando que la captura fue posiblemente desde la costa (Martinoli 2018). Por su parte, en la región del canal Beagle se propuso el ingreso de las carcasas completas a las áreas residenciales (con excepción de los animales de grandes dimensiones como los machos adultos de Otaria flavescens), con base en la presencia de tecnología de navegación y de arpones de punta desmontable que facilitarían la captura en el agua en momentos no reproductivos en el área de los canales interiores (Martinoli 2018; Orquera y Piana 1999; Schiavini 1990).

No obstante, pese a una diferencia en las abundancias anatómicas entre los conjuntos de los distintos sectores costeros, al correlacionar los valores de \%MAU con los de anatomía económica de la especie Arctocephalus australis (Martinoli 2019), en todos los casos las porciones más representadas son aquellas con utilidades entre moderadas y bajas (Figura 2), incluso en el Tercer Componente de Túnel I que indica una línea de tendencia positiva, pero un resultado de carácter negativo (Figura 2A). En Kaiyawoteha II (Figura 2C), además, la correlación entre los valores es negativa, fuerte y estadísticamente significativa $\left(r_{s}=-0,6 ; p<0,05\right)$. Por lo tanto, aunque es posible observar diferencias en la representación de los elementos, con una tendencia a la mayor completitud de las carcasas en la región del canal Beagle (Martinoli 2017, 2018), la relación entre las variables \%MAU y \%MUI es similar en los cuatro conjuntos aquí 
analizados; esto a pesar que se esperaba una selección de diferentes estrategias de aprovechamiento de las presas entre el canal Beagle central y Moat por las razones señaladas (Martinoli 2018).

\begin{tabular}{|c|c|c|c|c|c|c|c|c|c|c|c|c|}
\hline \multirow{3}{*}{ Unidades Anatómicas } & \multicolumn{9}{|c|}{ CANAL BEAGLE } & \multirow{2}{*}{\multicolumn{3}{|c|}{$\begin{array}{c}\text { MOAT } \\
\text { Heshkaia } 28\end{array}$}} \\
\hline & \multicolumn{3}{|c|}{ Túnel I (3er C) } & \multicolumn{3}{|c|}{ Túnel I (4to C) } & \multicolumn{3}{|c|}{ Kaiyawoteha II } & & & \\
\hline & NME & MAU & \%MAU & NME & MAU & \%MAU & NME & MAU & \%MAU & NME & MAU & \%MAU \\
\hline Mandíbula & 2 & 1 & 25 & 5 & 2,5 & 55,5 & 30 & 15 & 43,5 & 1 & 0,5 & 20 \\
\hline Atlas & 1 & 1 & 25 & 0 & 0 & 0 & 19 & 19 & 55,1 & 1 & 1 & 40 \\
\hline Axis & 4 & 4 & 100 & 0 & 0 & 0 & 12 & 12 & 34,8 & 0 & 0 & 0 \\
\hline V. Cervicales & 4 & 0,8 & 20 & 2 & 0,4 & 8,9 & 67 & 13,4 & 38,8 & 2 & 0,4 & 16 \\
\hline V. Torácicas & 11 & 0,7 & 17,5 & 8 & 0,5 & 11,1 & 185 & 12,3 & 35,6 & 5 & 0,3 & 12 \\
\hline V. Lumbares & 1 & 0,2 & 5 & 3 & 0,6 & 13,3 & 54 & 10,8 & 31,3 & 1 & 0,2 & 8 \\
\hline Sacro & 1 & 0,3 & 7,5 & 1 & 0,3 & 6,7 & 77 & 19,2 & 55,6 & 0 & 0 & 0 \\
\hline Costilla & 55 & 1,8 & 45 & 60 & 2 & 44,4 & 622 & 20,7 & 60 & 26 & 0,9 & 36 \\
\hline Esternebra & 2 & 0,25 & 6,3 & 8 & 1 & 22,2 & 133 & 16,6 & 49 & 2 & 0,2 & 8 \\
\hline Escápula & 2 & 1 & 25 & 1 & 0,5 & 11,1 & 23 & 11,5 & 33,3 & 0 & 0 & 0 \\
\hline Húmero & 4 & 2 & 50 & 6 & 3 & 66,7 & 38 & 19 & 55,1 & 2 & 1 & 40 \\
\hline Ulna & 3 & 1,5 & 37,5 & 8 & 4 & 88,9 & 30 & 15 & 43,5 & 1 & 0,5 & 20 \\
\hline Radio & 6 & 3 & 75 & 8 & 4 & 88,9 & 36 & 18 & 52,2 & 4 & 2 & 80 \\
\hline Articulares Ant. & 1 & 0,2 & 5 & 9 & 1,2 & 27 & 150 & 18,75 & 54,3 & 2 & 0,2 & 8 \\
\hline Metacarpianos & 14 & 1,4 & 35 & 13 & 1,3 & 29 & 191 & 19,1 & 55,4 & 5 & 0,5 & 20 \\
\hline Falanges Ants. Indet & 15 & 0,5 & 12,5 & 15 & 0,5 & 11,1 & 359 & 12,8 & 37,2 & 8 & 0,3 & 12 \\
\hline Pelvis & 3 & 1,5 & 37,5 & 9 & 4,5 & 100 & 58 & 29 & 84 & 2 & 1 & 40 \\
\hline Fémur & 5 & 2,5 & 62,5 & 4 & 2 & 44,4 & 69 & 34,5 & 100 & 1 & 0,5 & 20 \\
\hline Tibia & 4 & 2 & 50 & 3 & 1,5 & 33,3 & 43 & 21,5 & 62,3 & 5 & 2,5 & 100 \\
\hline Peroné & 3 & 1,5 & 37,5 & 5 & 2,5 & 55,5 & 38 & 19 & 55,1 & 3 & 1,5 & 60 \\
\hline Articulares Post. & 7 & 0,9 & 23 & 5 & 0,6 & 13,3 & 235 & 26,1 & 75,7 & 3 & 0,4 & 16 \\
\hline Metatarsianos & 10 & 1 & 25 & 10 & 1 & 22,2 & 231 & 23,1 & 67 & 2 & 0,2 & 8 \\
\hline Falanges Posts. Indet & 12 & 0,4 & 10 & 15 & 0,5 & 11,1 & 380 & 13,57 & 40 & 0 & 0 & 0 \\
\hline Total & 170 & - & - & 198 & - & - & 3080 & - & - & 76 & - & - \\
\hline
\end{tabular}

Tabla 1. Valores de NME, MAU y \%MAU correspondientes a pinnípedos en los conjuntos estudiados en la costas del sur de Tierra del Fuego.

Asimismo, al evaluar la correlación entre los valores de \%MAU de pinnípedos en cada uno de los conjuntos con los de densidad mineral ósea para Arctocephalus australis (Borella et al. 2007), al contrario de lo que ocurre con los valores de utilidad económica, los resultados son todos de carácter positivo (Figura 3) e incluso en el caso del Cuarto Componente de Túnel I y de Kaiyawoteha II son moderadamente fuertes y estadísticamente significativos. Asimismo, como se observa en los gráficos de dispersión (Figura 3B/C), es posible entonces sostener una tendencia general a la mayor preservación de aquellas porciones con alta densidad ósea y una pérdida de aquellos elementos con valores más bajos de densidad. Por lo tanto, la interpretación de los factores estructurantes de estos conjuntos se torna confusa en la medida en que comparamos las curvas de \%MAU/\%MUI y las de \%MAU/DMO. 

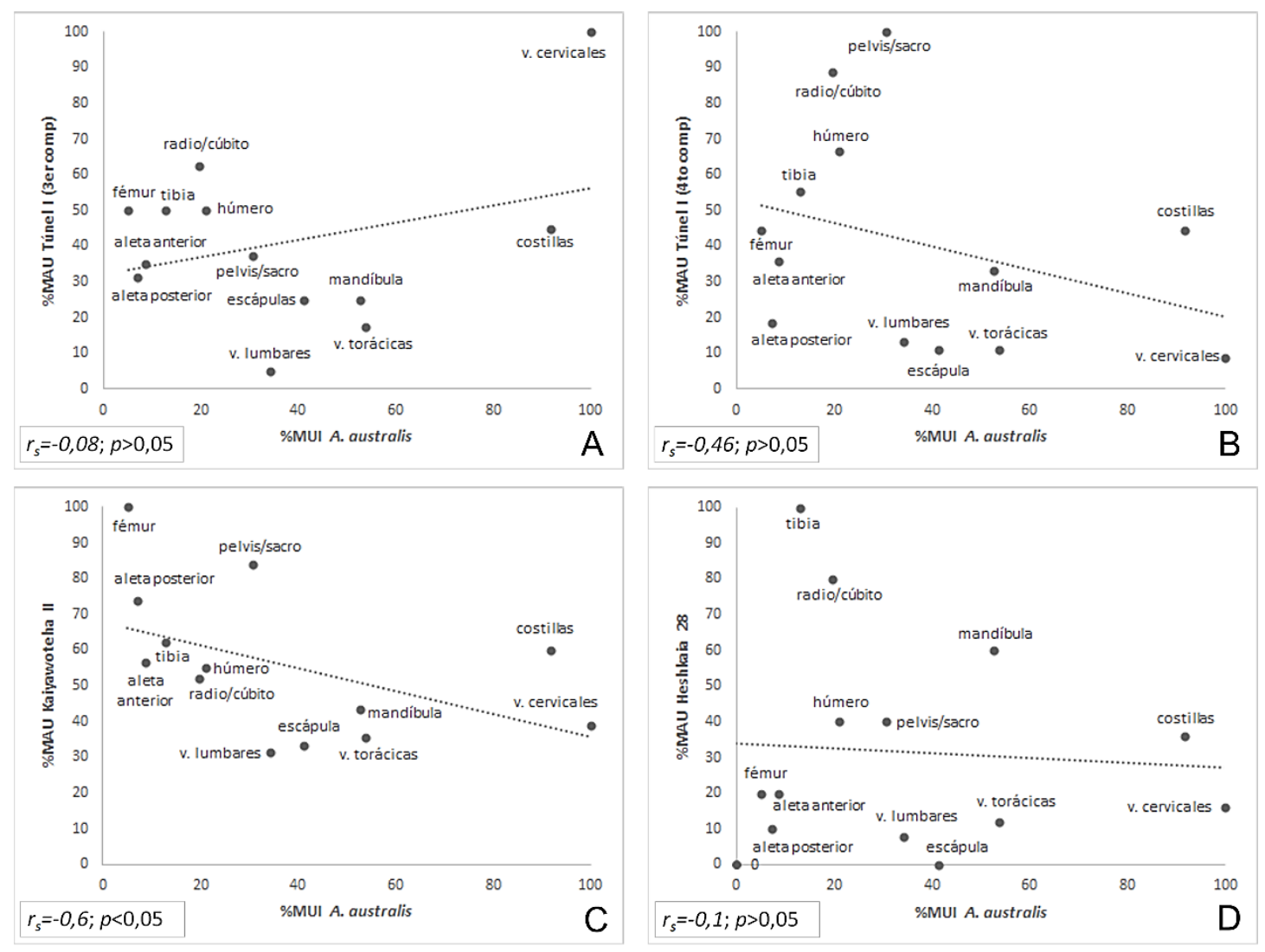

Figura 2. Gráficos de dispersión entre los valores de \%MUI para Arctocephalus australis (Martinoli 2019) y los valores de \%MAU de pinnípedos en: A) Tercer Componente de Túnel I;

B) Cuarto Componente de Túnel I; C) Kaiyawoteha II y D) Heshkaia 28.

Algunas consideraciones sobre la densidad mineral ósea en otáridos y las expectativas de supervivencia diferencial

Uno de los aspectos más debatidos en relación con la interpretación de los perfiles anatómicos observados en conjuntos zooarqueológicos es aquel relacionado con la equifinalidad. Tal problemática surge a partir de la interdependencia (aunque no necesariamente la correlación) entre los índices de utilidad económica y los valores de densidad mineral ósea en varias especies de mamíferos terrestres; relación que en el caso de los guanacos (Lama guanicoe) es incluso fuerte y estadísticamente significativa (Lyman 1994: 259). Respecto de los pinnípedos, se ha propuesto que su mayor densidad ósea general reduciría dicho problema (Lyman et al. 1992). Sin embargo, al correlacionar los valores de densidad mineral ósea (Borella et al. 2007) ${ }^{1}$ para la especie Arctocephalus australis (la más abundante en los conjuntos zooarqueológicos de la región del canal Beagle) con el índice de utilidad económica recientemente calculado para dicha especie (Martinoli 2019), observamos una relación muy similar a la hallada en el caso de muchos mamíferos terrestres (Lyman 1994). Es decir, aunque no es posible establecer una relación causal $\left(r_{s}=-0,1 ; p>0,05\right)$, el gráfico de dispersión muestra cierta correspondencia entre las variables (Figura 4): las porciones con menor densidad ósea tienden a tener valores de utilidad económica más elevados, mientras que las de menor utilidad presentan una densidad ósea mayor. Es decir, de acuerdo con la 
propuesta de Lyman (1994), las curvas de utilidad económica negativas relevadas en los conjuntos de la costa sur de Tierra del Fuego (Martinoli 2018) podrían reflejar la supervivencia diferencial de unidades anatómicas por sus valores de densidad mineral ósea y/o el transporte/descarte de porciones de bajo rendimiento.
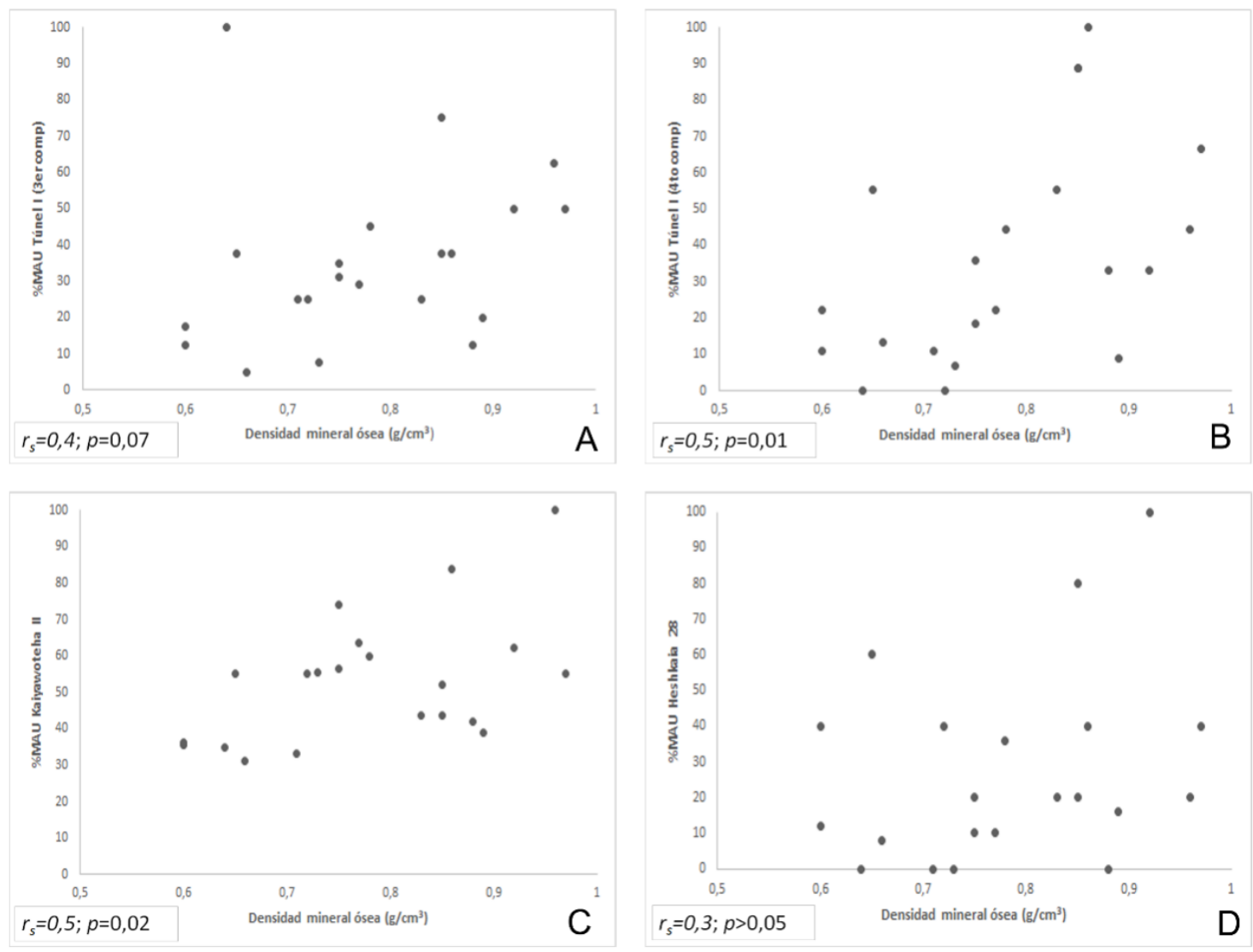

Figura 3. Gráficos de dispersión entre los valores de densidad mineral ósea para A. australis (Borella et al. 2007) y los valores de \%MAU de pinnípedos en: A) Tercer Componente de Túnel I;

B) Cuarto Componente de Túnel I; C) Kaiyawoteha II y D) Heshkaia 28.

En términos generales, por un lado, la DMO puede interactuar con procesos postdepositacionales tales como la meteorización, ya que dicho fenómeno se relaciona, en parte, con la disolución, recristalización y alteración química de la fracción mineral de los huesos (Behrensmeyer 1978). Por otro lado, los huesos con menor DMO pueden presentar índices de fragmentación más elevados. Por lo cual, como ya han mostrado los seguimientos realizados en acumulaciones naturales de carcasas de pinnípedos en sectores costeros de Patagonia, se observa un mayor índice de fragmentación y/o daño en huesos de baja densidad mineral ósea como vértebras torácicas y lumbares, escápulas y costillas (Borella y Muñoz 2006; Muñoz 2008). Por lo que tales elementos pueden presentar índices bajos de identificación, incluso estar ausentes en los conjuntos si consideramos la extensión del daño (Lyman 1994).

Ahora bien, por la ausencia de canal medular en los huesos largos, en la generalidad, los huesos de pinnípedos han tendido a ser descartados enteros, lo que implica que los eventuales carroñeros podían acceder de manera secundaria a huesos 
completos, los que conservaban parte de su valor nutricional (Muñoz 2009). Lo dicho es de relevancia para nuestro planteo, puesto que los elementos con menor DMO son preferidos por los carnívoros, por ser relativamente más fácil el acceso a la grasa contenida en el tejido trabecular (Marean y Cleghorn 2003).

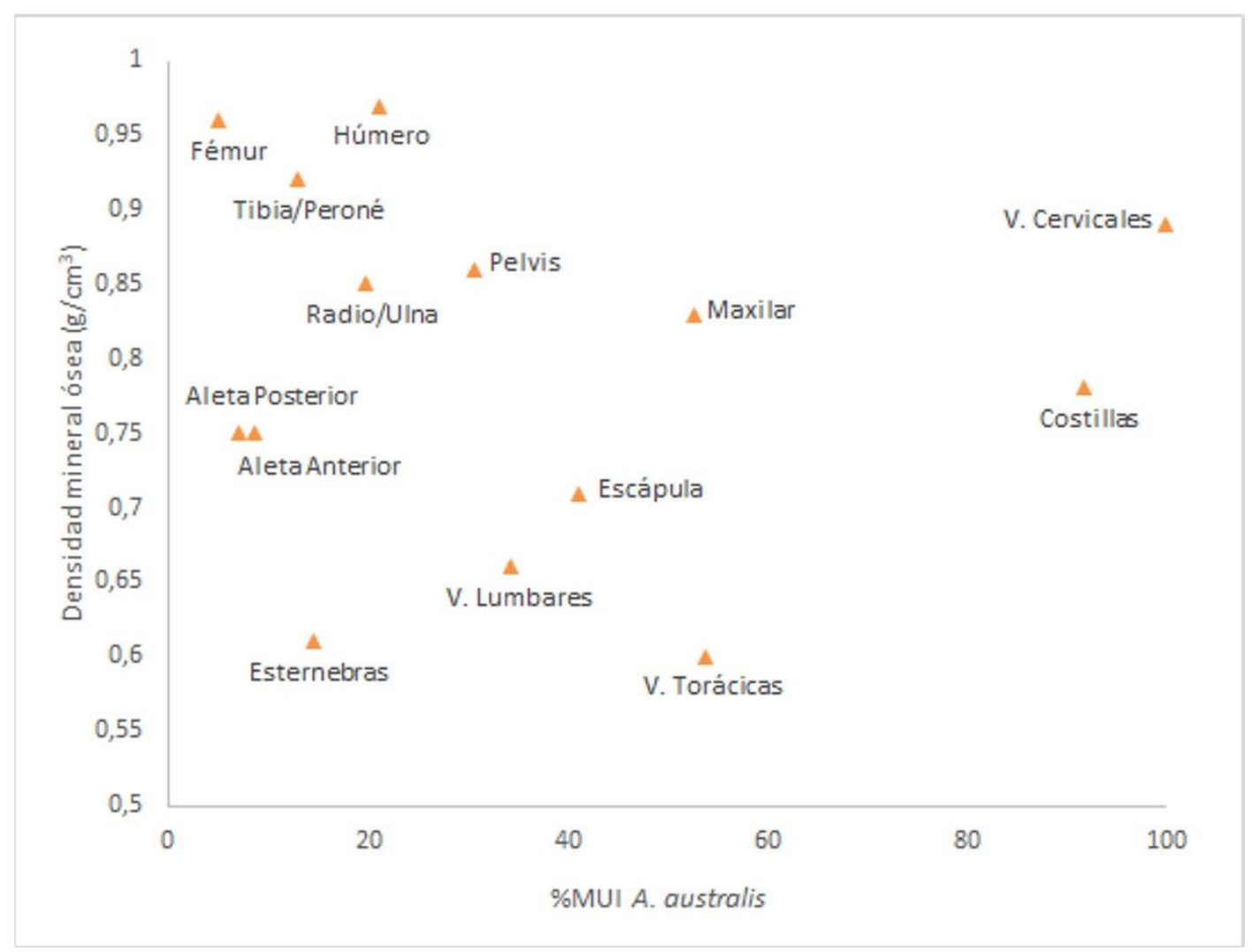

Figura 4. Gráfico de dispersión entre los valores de densidad mineral ósea de Arctocephalus australis (Borella et al. 2007) y los valores de utilidad económica (\%MUI) para Arctocephalus australis (Martinoli 2019).

Los carnívoros actúan sobre los restos faunísticos de dos maneras distintas: por un lado, generan daños que a veces pueden dificultar la identificación de las unidades anatómicas; por otro lado, transportan/sustraen/acumulan elementos (Brain 1981). En el caso de Tierra del Fuego se ha propuesto que el daño infligido por estos agentes fue escaso por dos motivos: a) los carnívoros disponibles en la región tienen un bajo poder destructivo (Muñoz 2009); y b) la disponibilidad general de carcasas a lo largo del Holoceno habría sido relativamente alta, por lo que no habría existido competencia para su consumo (Borrero 1988, 1990). En lo que respecta a animales depredadores que puedan modificar los perfiles anatómicos, en distintos sectores de Patagonia fue documentada la acción de aves y cánidos sobre carcasas completas y sobre huesos aislados (Estévez y Mameli 2000; Cruz 2003, 2015; Martin 1998; Vázquez et al. 2016). Específicamente, tales observaciones han indicado que los primeros elementos que se pierden por la actividad de los carroñeros son los pequeños, tarsianos y carpianos, cuando no se encuentran articulados y con tejido blando aún adherido (Borella y Borrero 2010; Borella y Muñoz 2006; Cruz 2003, 2015). Lo mismo podría ser pertinente para el caso de falanges y metapodios, los cuales posiblemente no pueden ser 
deglutidos enteros por especies carroñeras existentes en Tierra del Fuego, aunque pueden ser transportados por zorros (Estévez y Mameli 2000; Martin 1998) y algunas especies de aves (Vázquez et al. 2016).

Entonces, aunque es bien conocido el efecto de decaimiento diferencial de elementos anatómicos por sus valores de $\mathrm{DMO}$, tal factor también puede interactuar con otros procesos y agentes, como los carnívoros. Consideramos que, metodológicamente, sería posible determinar un umbral a partir del cual los restos óseos de pinnípedos tienen más probabilidades de sobrevivir en contextos arqueológicos de la costa sur de Tierra del Fuego y podría ser definido mediante dos variables de carácter interdependiente (Marean y Cleghorn 2003): los valores de DMO y la acción de los carroñeros (en función del tamaño de los elementos). Para definir los rangos de tamaño hemos utilizado como referencia los valores de largos totales promediados correspondientes a la especie Arctocephalus australis porque es la más abundante en los conjuntos zooarqueológicos de la región (Martinoli 2018, 2019; Orquera y Piana 1999; Schiavini 1990). Como se observa en la Figura 5, las variables muestran una relación general positiva que se corrobora con el resultado del análisis de correlación $\left(r_{s}=0,57 ; p=0,01\right)$, el cual es moderadamente fuerte y estadísticamente significativo: cuanto más grande es el elemento anatómico mayor es la densidad ósea registrada. La decisión de tomar como parámetro el largo total es exclusivamente metodológico, el objetivo es establecer algún tipo de relación general que nos permita comenzar a generar métodos de análisis específicos para los conjuntos óseos de pinnípedos.

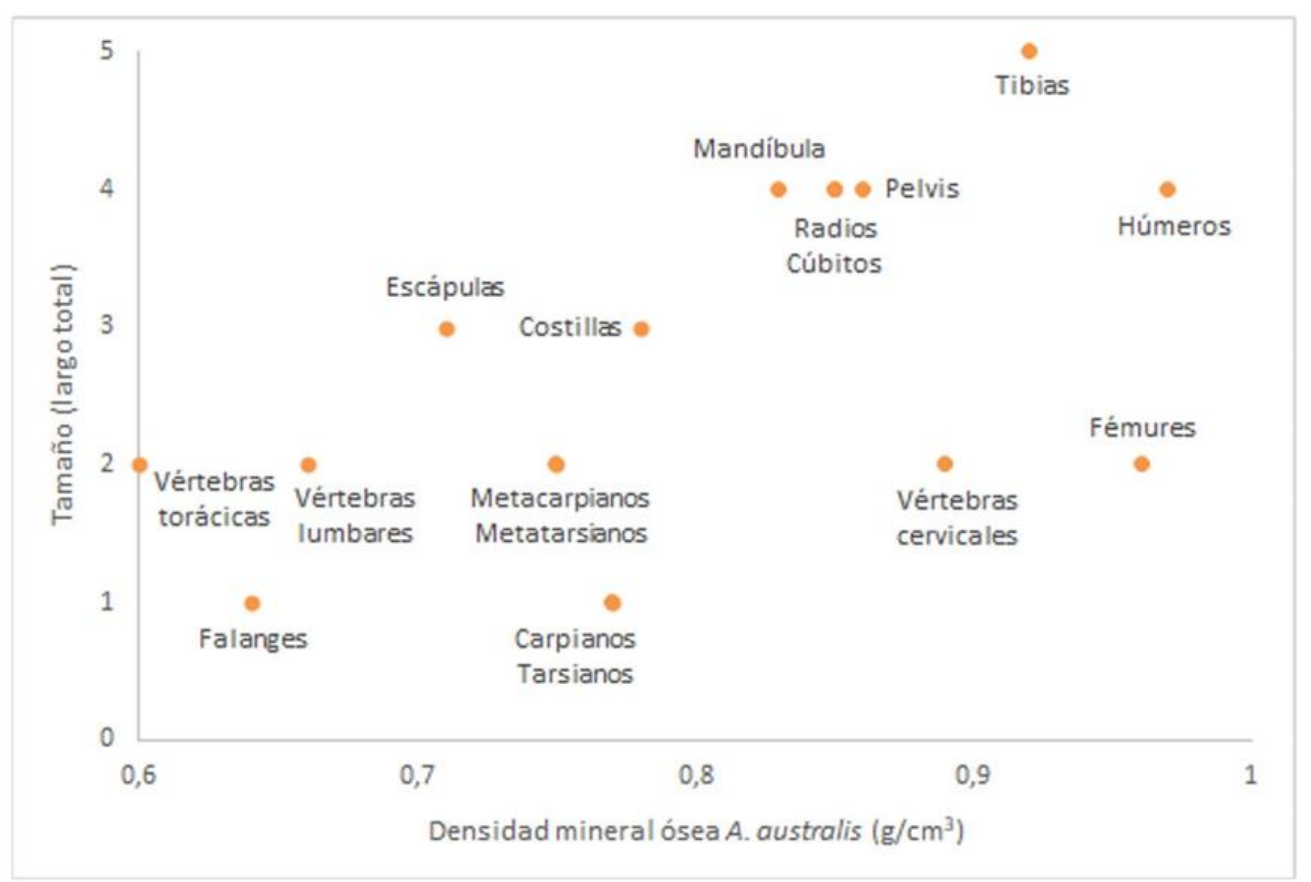

Figura 5. Gráfico de correlación entre los valores de densidad mineral ósea (Borella et al. 2007) y los tamaños promedio (largos totales) de los huesos largos de Arctocephalus australis macho adulto. Las categorías de tamaño fueron definidas en intervalos de $5 \mathrm{~cm}(1=0$ a $5 \mathrm{~cm} ; 2=5$ a 10 $\mathrm{cm} ; 3=10$ a $15 \mathrm{~cm} ; 4=15$ a $20 \mathrm{~cm} ; 5=20$ a $25 \mathrm{~cm}$ ). 
Al hallar una relación significativa entre los largos totales y los valores de DMO, consideramos que es posible usar esta última como indicador adecuado del potencial de supervivencia de las distintas unidades anatómicas que conforman el esqueleto de los pinnípedos. Siguiendo la propuesta de Marean y Cleghorn (2003) agrupamos en dos conjuntos discretos las partes esqueletarias: los de alto potencial y los de bajo potencial de supervivencia (Figura 6). Seleccionamos $0,8 \mathrm{~g} / \mathrm{cm}^{3}$ como valor discriminante entre ambos grupos, en función de las observaciones actualísticas detalladas más arriba. En términos generales, los restos óseos con valores de densidad mineral ósea por debajo de ese valor mostraron mayor incidencia de agentes tafonómicos de origen no antrópico (Borella y Borrero 2010), incluso aquellos que tienen tamaños medios como costillas y escápulas.

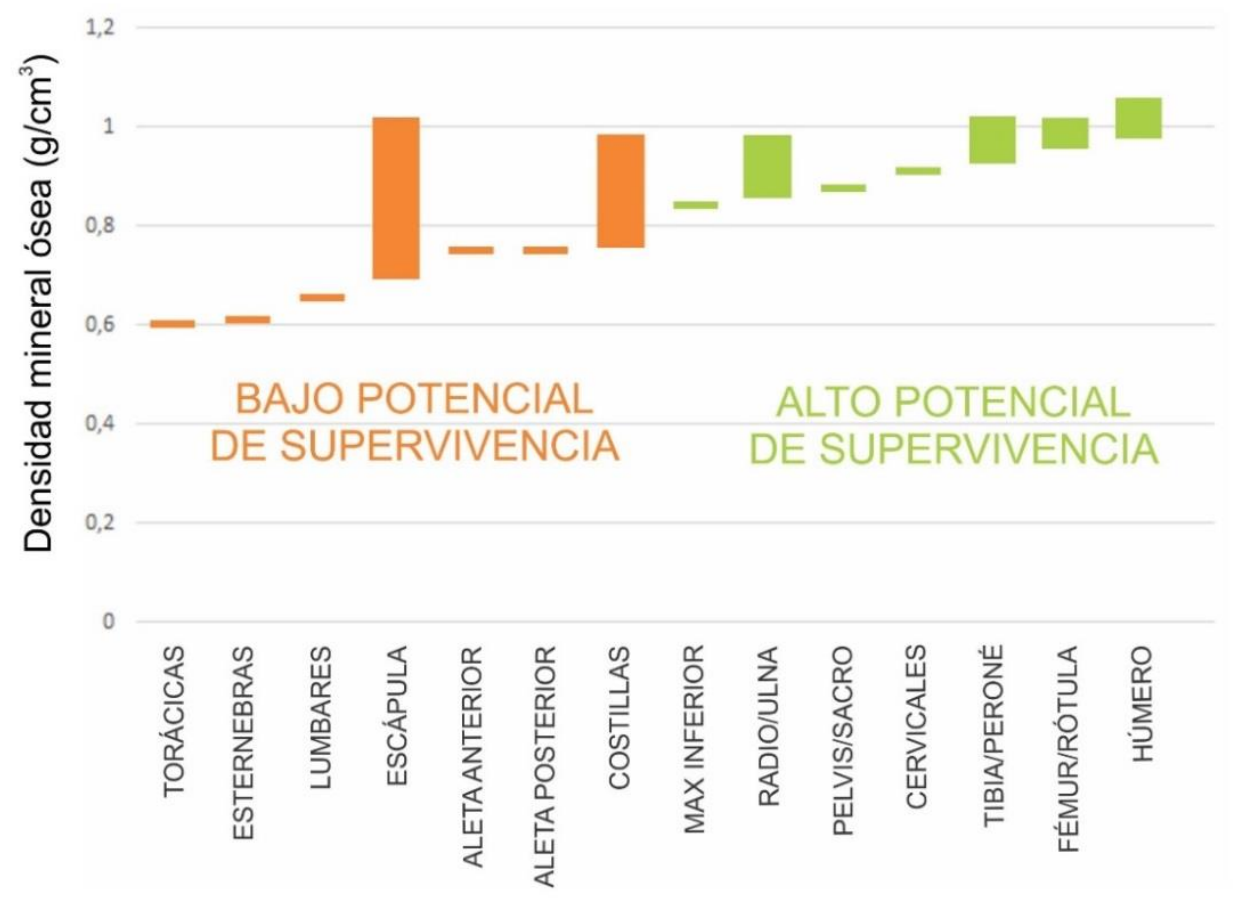

Figura 6. Definición de los conjuntos de alto y bajo potencial de supervivencia en función de los valores de densidad mineral ósea (Borella et al. 2007).

La propuesta de agrupar los restos óseos de pinnípedos en dos conjuntos según su potencial de supervivencia permitiría evaluar de manera separada sus trayectorias de preservación. Si el conjunto óseo no sufrió procesos postdepositacionales que alteraron su estructura, ambos grupos deberían comportarse de modo similar, es decir indicarían curvas comparables entre sí. Por el contrario, en la medida en que los grupos de bajo y alto potencial de supervivencia se manifiesten con formas opuestas, es posible que hayan actuado de manera más o menos extensiva procesos que enmascaren y dificulten la identificación de las actividades culturales que originaron tales conjuntos faunísticos. Esta propuesta de análisis, además, nos permitiría evaluar las posibles estrategias de transporte, ya que, aunque la relación entre la densidad mineral ósea y los valores de utilidad económica muestran una relación general negativa, al evaluar 
los valores de utilidad económica en función de los grupos propuestos previamente es posible realizar una observación. En concordancia con lo dicho, la mayoría de las unidades anatómicas que integran el primer grupo presentan valores de \%MUI menores o iguales al $30 \%$, mientras que la mayor parte de los elementos que integran el grupo de bajo potencial de supervivencia tienen valores de rendimiento por encima de ese porcentaje (Figura 7). Sin embargo, en ambos grupos hallamos elementos de bajo, moderado y alto valor económico. Lo dicho implica que el conjunto de alto potencial de supervivencia podría utilizarse para la evaluación de pautas de transporte cuya variable de selección sea el rendimiento económico de las porciones (Marean y Cleghorn 2003).

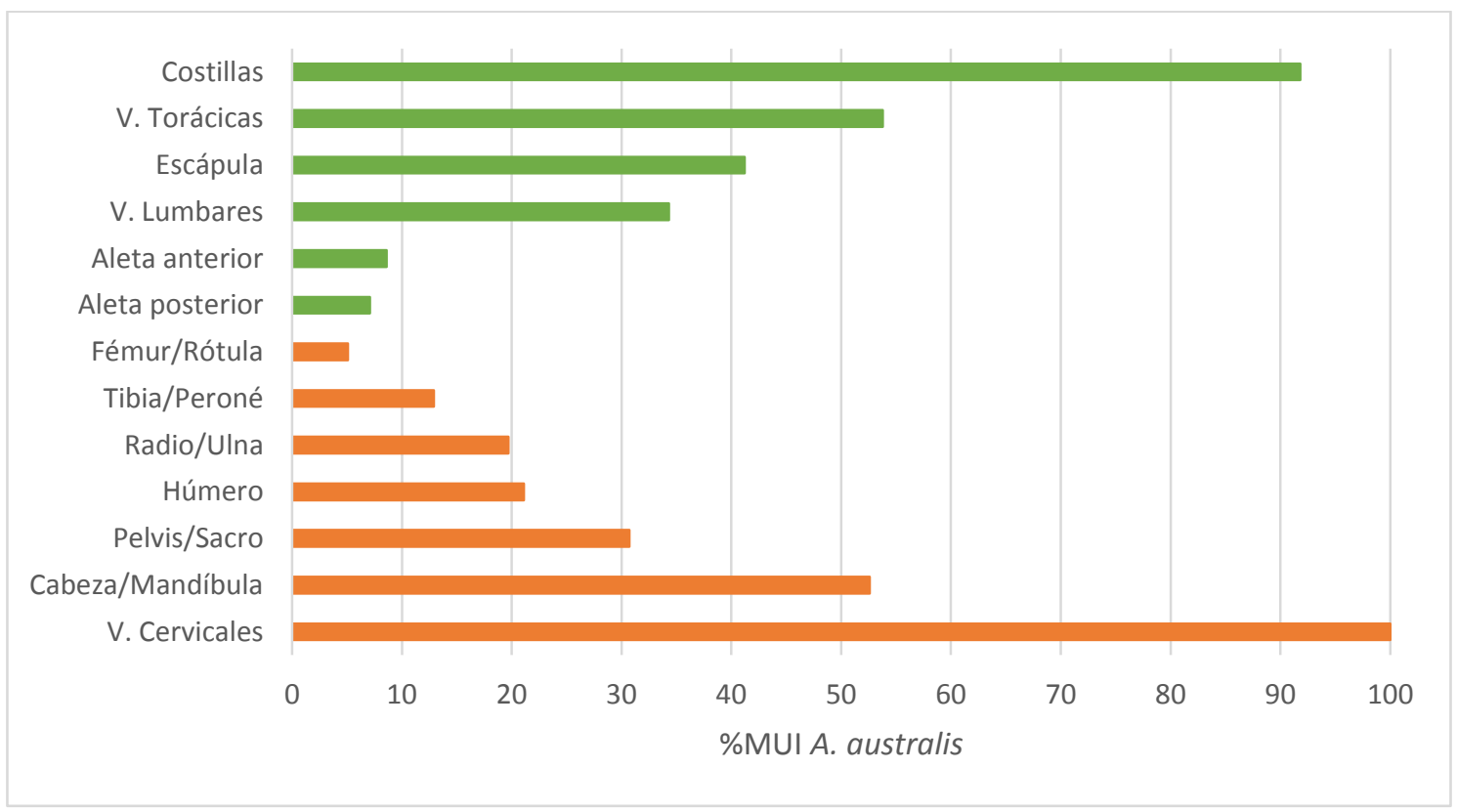

Figura 7. Distribución de los valores de utilidad económica (\%MUI) para Arctocephalus australis (Martinoli 2019) en los conjuntos de alto y bajo potencial de supervivencia definidos en el presente trabajo.

\section{Resultados}

$\mathrm{Si}$ consideramos algunas observaciones sobre las modificaciones óseas documentadas, los conjuntos correspondientes a Túnel I presentan frecuencias similares en variables tafonómicas tales como fractura, termoalteración y meteorización, la cual no supera al estadio 2 (Figura 8). No obstante, los dos Componentes analizados en este yacimiento muestran una relación distinta entre las frecuencias anatómicas y los valores de DMO. Por un lado, aunque es posible destacar una alta incidencia de fracturas de origen natural en ambos conjuntos, éstas no parecen haber actuado sobre el grado de identificación de los elementos en el Tercer Componente de Túnel I, ya que se han determinado a nivel anatómico el $100 \%$ de los restos óseos correspondientes a pinnípedos. Por otro lado, un aspecto que caracteriza al Cuarto Componente de Túnel, en comparación con otros conjuntos de pinnípedos de la región del canal Beagle estudiados previamente (Martinoli 2018), es la presencia de 
marcas de raíces de especies vegetales (NISP\%=1,5), las que pueden haber afectado de manera más extensiva la integridad de este conjunto, reflejado en un índice de fragmentación mayor (Figura 8). Si damos cuenta del proceso de formación de estos depósitos, es posible observar que presentan ciertas diferencias, las cuales son aspectos a considerar para comprender la incidencia de procesos tafonómicos. El Tercer Componente es un depósito homogéneo que corresponde únicamente a la capa C3 (cuadrícula XIII), que es un nivel limoso con alto contenido de materia orgánica (Orquera y Piana 1999). Por el contrario, el Cuarto Componente es de carácter más heterogéneo, ya que incluye a la capa C2 (suelo) y a dos lentes de concheros Gama y Alfa (Orquera y Piana 1999). Si bien en el presente trabajo la mayoría de los restos óseos analizados provienen de la lente Alfa, algunos de los huesos que muestran marcas de radículas se encontraban en contacto con la capa C2. Por lo tanto, en el caso del Cuarto Componente es posible que se estén promediando conjuntos que han tenido tiempos y modos distintos de formación, con diferencias en la incidencia de algunos procesos postdepositacionales, lo cual puede haber influido en los perfiles anatómicos.

Por su parte, en Kaiyawoteha II sólo dos especímenes presentaron marcas de carnívoros $(\mathrm{NISP} \%=0,02)$, pero hay una incidencia elevada de meteorización en comparación con los otros conjuntos de pinnípedos estudiados, aunque en estadios bajos (Figura 8): el 64 \% de los restos óseos se encuentran en los estadios 1 y 2 (sensu Behrensmeyer 1978). No obstante, el informe de excavación (Piana et al. 2007) no da cuenta de rasgos en los depósitos que puedan indicar procesos de entierro y reexposición del material. En términos generales, los concheros se caracterizan por una acumulación rápida y por ser sustratos químicamente estables (Orquera y Piana 1992, 2001:250). No obstante, en Kaiyawoteha II una parte significativa del material procede de un suelo donde se intercalaban diferentes acumulaciones de conchal (Piana et al. 2007). Es decir, que el proceso general de sepultamiento del material en este sitio puede haber sido lento, considerando que la sedimentación respondió al lapso de formación del suelo por encima del depósito de origen antrópico, con una tasa de acumulación más lenta que la de los concheros. Asimismo, se observa un porcentaje significativo $(\mathrm{NISP} \%=17,1)$ de especímenes con termoalteración (quemados), lo que pudo influir en el grado de fragmentación y, en consecuencia, en la cantidad de restos óseos de pinnípedos indeterminados a nivel anatómico (Figura 8).

En Heshkaia 28, no han podido ser identificados un total de 4,5\% restos óseos a nivel anatómico (Figura 8). Comparativamente, una de las observaciones que resalta es la elevada incidencia de marcas de carnívoros, dado que el $4 \%$ de los especímenes presentan este tipo de modificaciones. Estas alteraciones incluyen perforaciones, ahuecamientos y bordes crenulados compatibles con la acción de cánidos (Martinoli 2018). Los elementos afectados corresponden a: radio, cúbito, pelvis, escafolunar y costilla. A su vez, en Heshkaia 28 fue identificado un amplio sector con sedimento alterado térmicamente (Martinoli 2018), y un porcentaje alto de especímenes quemados $(\mathrm{NISP} \%=16,9)$ y calcinados $(\mathrm{NISP} \%=26,5)$. 


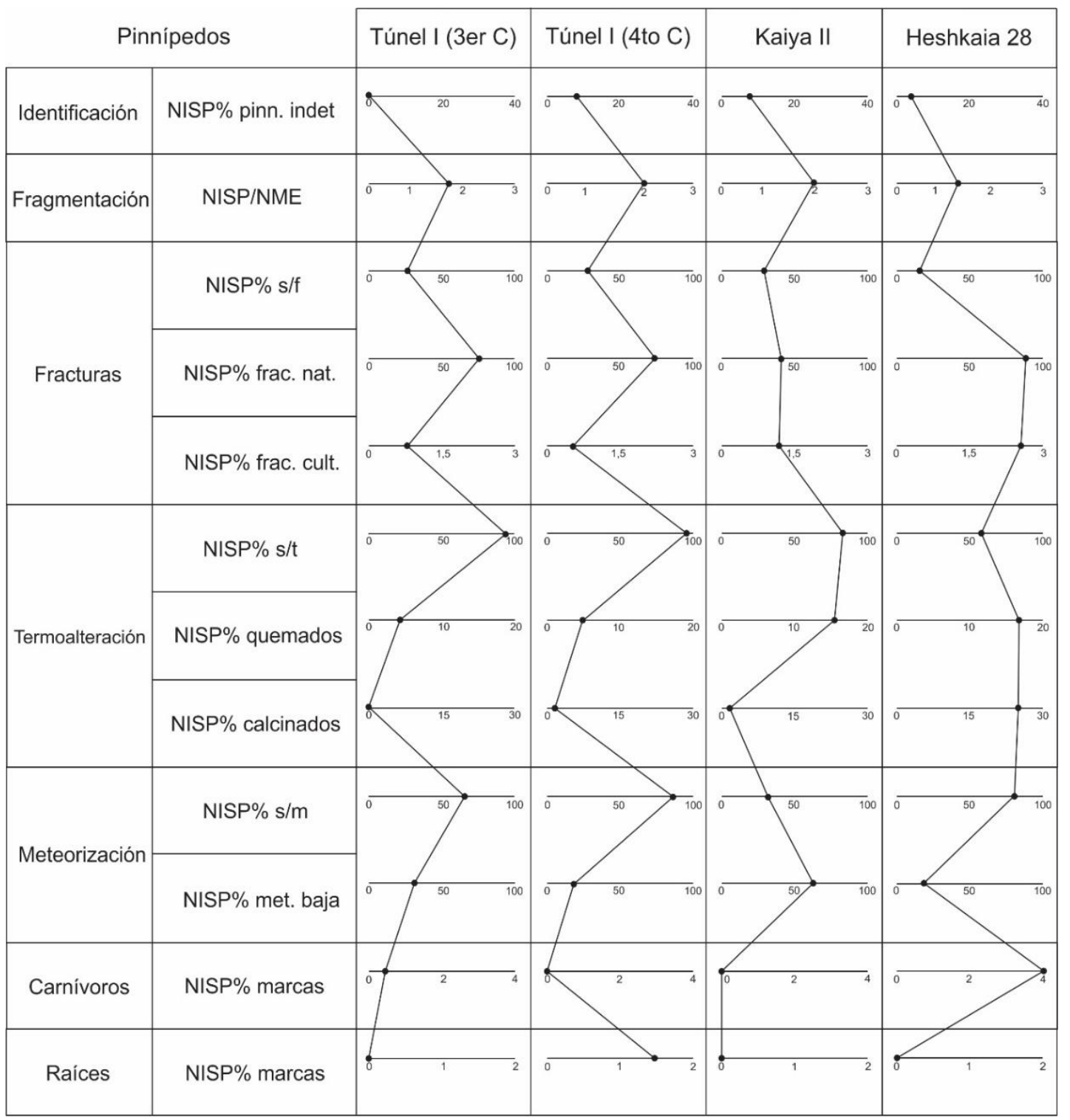

Figura 8. Tafograma comparativo de los conjuntos de pinnípedos analizados de la costa sur de Tierra del Fuego.

Grupos de alto y bajo potencial de supervivencia

El primer caso examinado es el Tercer Componente del sitio Túnel I. Como se puede observar en la Figura 9A, la evaluación por separado de ambos conjuntos indica una tendencia muy similar y en ambos casos el resultado es muy cercano a cero y estadísticamente no significativo. Dicho resultado es prácticamente idéntico al obtenido a partir de la evaluación global de las abundancias anatómicas. Dada la similitud entre ambas tendencias, es posible que en este sitio las propiedades óseas intrínsecas como la densidad mineral ósea, no hayan jugado un rol central en la preservación de los conjuntos óseos de pinnípedos. De todos modos, al centrarnos específicamente en el conjunto con bajo potencial de supervivencia, es posible advertir que todos los elementos que conforman dicho grupo se encuentran representados en porcentajes menores al $50 \%$, incluso muchos de ellos por debajo del $20 \%$. Por su parte, 
en términos generales, los huesos largos y las vértebras cervicales son las unidades anatómicas relativamente más abundantes, pero sólo este último elemento parece guardar una relación entre su abundancia y su rendimiento.

En el Cuarto Componente de Túnel I, el análisis de correlación global indicó un resultado negativo, moderadamente fuerte y estadísticamente no significativo. Asimismo, los valores de \%MAU y los de densidad mineral ósea mostraron un resultado positivo, moderadamente fuerte y estadísticamente significativo. Al evaluar por separado los conjuntos de alto y bajo potencial de supervivencia, se obtiene un resultado interesante: a diferencia del Tercer Componente, en este conjunto las tendencias son casi opuestas (Figura 9B). En el caso del conjunto con alto potencial de supervivencia la correlación es negativa y estadísticamente no significativa, mientras que en el de bajo potencial de supervivencia el resultado es cercano a cero y la tendencia es levemente ascendente. A su vez, todos los elementos que conforman este último grupo están representados por porcentajes menores al $50 \%$.
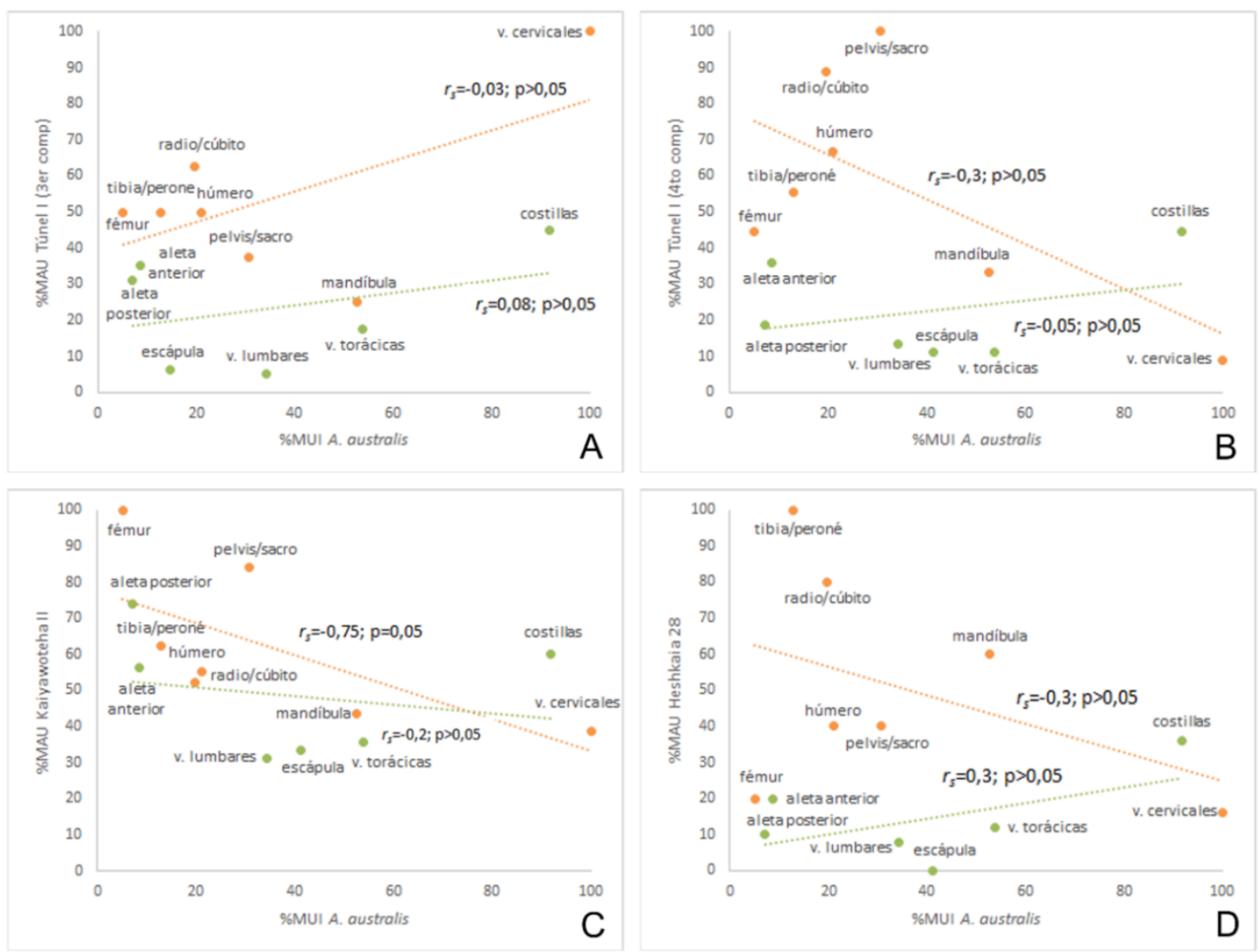

Figura 9. Gráficos de dispersión, diferenciando los conjuntos de alto y bajo potencial de supervivencia, entre los valores de \%MUI de Arctocephalus australis (Martinoli 2019) y los valores de \%MAU de pinnípedos en: A) Tercer Componente de Túnel I; B) Cuarto Componente de Túnel I; C) Kaiyawoteha II y D) Heshkaia 28. 
El sitio Kaiyawoteha II es el único que indicó una correlación global negativa, moderadamente fuerte y estadísticamente significativa entre los valores de rendimiento económico y las abundancias anatómicas, a la vez que un resultado positivo, moderadamente fuerte y estadísticamente significativo entre los valores de densidad mineral ósea y los de \%MAU. Es decir, estos resultados son indicativos de un claro fenómeno de equifinalidad entre distintos procesos, como el señalado por Lyman (1994: 259). Pues bien, al evaluar por separado los conjuntos de alto y bajo potencial de supervivencia, observamos que las tendencias muestran diferencias entre sí (Figura 9C). Mientras el conjunto de alto potencial de supervivencia señala una clara tendencia negativa fuerte y estadísticamente significativa, el conjunto de bajo potencial de supervivencia indica un valor menos negativo y una tendencia más difusa entre los valores.

El último sitio analizado corresponde a Heshkaia 28, ubicado en la zona de Moat. El análisis de correlación entre los valores \%MAU del conjunto global y los de \%MUI indicó una relación negativa muy débil y estadísticamente no significativa, pero con una leve tendencia a la mayor presencia de elementos de bajo/moderado valor económico. Ahora bien, al considerar por separado los conjuntos de alto y bajo potencial de supervivencia tanto los resultados de las correlaciones, como las tendencias observadas son exactamente opuestas (Figura 9D). El conjunto de alto potencial de supervivencia indica una tendencia negativa, siendo los huesos largos los elementos más abundantes y los que presentan valores de rendimiento más bajos. En el caso de las unidades anatómicas que conforman el conjunto de bajo potencial de supervivencia, aunque todas están presentes en porcentajes menores al $40 \%$, muestran una tendencia de carácter positiva, aunque estadísticamente no significativa.

\section{Discusión}

Los resultados del análisis presentado indican que los conjuntos óseos de pinnípedos presentan estados de preservación diferencial. En primer lugar, dicho fenómeno se identifica al comparar entre sí los conjuntos de los diferentes sitios analizados y, en segundo lugar, al interior de los conjuntos según los perfiles de partes esqueletarias esperados en función de su potencial de supervivencia. Según estos resultados, el perfil anatómico registrado en el Tercer Componente de Túnel I es el menos afectado por factores tafonómicos, ya que las tendencias entre los conjuntos de alto y bajo potencial de supervivencia son prácticamente iguales, lo cual es compatible con una presencia baja de modificaciones óseas. En cambio, en los tres conjuntos restantes las tendencias observadas presentan variaciones, especialmente en el Cuarto Componente de Túnel I y en Heshkaia 28, que indican que los perfiles anatómicos pudieron haber estado expuestos a procesos naturales en diferente grado.

Un aspecto a considerar en la formación de estos conjuntos es la posibilidad de mezcla entre materiales arqueológicos y restos depositados con posterioridad, factor que ha sido observado en distintos espacios de la región costera de Tierra del Fuego- 
Patagonia (Alunni et al. 2017; Borella y Borrero 2010, 2019; Borella y Muñoz 2006; Borrero 2004; Muñoz 2008). En términos generales, los huesos de pinnípedos hallados en playas son principalmente el resultado de la deriva de animales por corrientes marinas y el posterior proceso de desarticulación y redepositación (Borrero 2004). De todos modos, no debe subestimarse el transporte y acumulación que pueden realizar los carnívoros (Muñoz 2009). En efecto, dada la distancia de los sitios aquí analizados a la línea de costa actual (150 m como máximo), en conjunto con la baja integridad anatómica observada especialmente en Heshkaia 28, no es posible descartar que este tipo de aportes haya ocurrido en los sitios analizados (Borella y Borrero 2010; Borrero 2004; Muñoz 2008). En este sentido, algunos trabajos actualísticos recientes han desarrollado una serie de observaciones para caracterizar las acumulaciones naturales de huesos de pinnípedos en contextos de playa. En primer lugar, los restos óseos recolectados en espacios costeros muestran niveles de meteorización heterogéneos, abarcando desde el estadio 1 al 4, lo cual contrasta con la meteorización registrada en conjuntos de origen antrópico, más homogénea, donde no se supera el estadio 2 (Borella y Muñoz 2006). Esto último es lo que caracteriza a los conjuntos faunísticos aquí presentados (Figura 8). A su vez, se observan estadios de meteorización diferencial entre la parte superior e inferior del elemento y/o la presencia de líquenes en la superficie expuesta, lo que tampoco se ha observado en los restos óseos analizados. Las acumulaciones naturales de restos de pinnípedos también se diferencian en términos de integridad anatómica y categorías de edad con respecto a los conjuntos arqueológicos (Borella y Borrero 2010, 2019; Borrero 2004; Muñoz 2008). Una de las observaciones de Muñoz (2008) es que las acumulaciones naturales recuperadas en dos sectores de la costa atlántica fueguina muestran una representación muy baja de individuos inmaduros, los cuales son relativamente abundantes en muchos de los sitios arqueológicos de dicha región, al igual que en la costa sur (Martinoli 2018; Schiavini 1990) y en otras costas patagónicas (Borella y Borrero 2010; Cruz et al. 2015; Legoupil 1989). Asimismo, en el caso de muertes naturales ocurridas en ámbitos de loberías $\mathrm{y} / \mathrm{u}$ osamentas que han sufrido procesos de momificación (Borrero 2004), la integridad anatómica observada es mucho mayor respecto de los conjuntos arqueológicos e incluso los huesos pueden aparecer articulados (Muñoz y Cruz 2014; Serrán et al. 2008). Esto también contrasta con los restos zooarqueológicos aquí analizados, en los cuales se reconoce desarticulación coherente con el consumo de las presas, además de marcas de corte relacionadas con el procesamiento de las presas (Martinoli 2017, 2018).

En lo que refiere a la interdependencia entre los elementos del conjunto de alto potencial de supervivencia y el \%MUI en canal Beagle central, pueden darse distintas interpretaciones. Tanto en el Tercer Componente como en el Cuarto Componente de Túnel I, los resultados de las correlaciones no son estadísticamente significativos, incluso en el primer conjunto el resultado es cercano a cero. Por lo cual, el rendimiento económico no fue una variable de selección para el transporte de partes esqueletarias. Especialmente si consideramos la disponibilidad de medios de navegación, es posible suponer que los costos de transporte no fueron elevados (Ames 2002), lo que puede 
haber implicado que los pinnípedos hayan sido transportados completos a las áreas residenciales y allí procesados para su consumo. Sin embargo, en el caso de Kaiyawoteha II la tendencia descripta en los resultados marca una diferencia con respecto a Túnel I, ya que el conjunto de alto potencial de supervivencia arroja como resultado una correlación negativa fuerte y estadísticamente significativa, sin olvidar que es posible definir un gradiente de elementos de bajo, moderado y alto valor de utilidad económica dentro de ese grupo (Marean y Cleghorn 2003). Para avanzar sobre la interpretación de este resultado es necesario resaltar dos aspectos. Por un lado, si bien no es posible descartar la mezcla de materiales, no hemos podido hallar en el conjunto indicios de dicho fenómeno: los niveles de meteorización son homogéneos, no se han encontrado elementos de pinnípedos en posición articulada (Piana et al. 2007) y los perfiles de mortalidad presentan individuos juveniles y subadultos en porcentajes significativos (Martinoli 2018). Por otro lado, existe la posibilidad que las porciones de alto potencial de supervivencia también hayan sufrido procesos de pérdida al considerar los elevados niveles de termoalteración que se observan en Kaiyawoteha II. Por lo tanto, aunque el perfil anatómico permitiría proponer la puesta en práctica de una estrategia de transporte/descarte de porciones de bajo rendimiento, es necesario ser cautelosos con su interpretación, dados los niveles de alteración térmica.

En el caso de Heshkaia 28 es posible que agentes postdepositacionales hayan alterado las abundancias anatómicas. En primer lugar, las que corresponden al grupo de bajo potencial de supervivencia, pueden haber sido afectados por la acción de carnívoros a partir de la sustracción de elementos pequeños, así como la destrucción de aquellos con baja densidad. En segundo lugar, es posible que la acción térmica haya generado pérdida en la capacidad de identificación a nivel esqueletario y taxonómico. Por lo tanto, a la luz de los resultados obtenidos en este trabajo, las interpretaciones realizadas previamente sobre un transporte selectivo de porciones de bajo/moderado valor económico de pinnípedos medianos y pequeños a Heshkaia 28 debe ser reconsiderada (Martinoli 2018). No obstante, la integridad anatómica en este conjunto fue más baja que la observada en la región del canal Beagle. En efecto, en la zona de Moat los pinnípedos fueron presas eventuales, probablemente capturadas de manera oportunística en sectores de playa (Martinoli 2018), por lo que la densidad de sus restos óseos en el sitio arqueológico debe haber sido escasa. Posiblemente en este espacio costero los carnívoros ejercieron una competencia mayor (Borrero 2004) por el acceso a la grasa contenida en el tejido trabecular de los huesos. Con lo cual, es factible que los procesos postdepositacionales sean arqueológicamente más visibles, dado un volumen menor de restos óseos de pinnípedos.

\section{Conclusión}

Los resultados aquí presentados indican perfiles anatómicos variables aún para contextos donde se presume la incorporación de carcasas completas de pinnípedos en los sitios. A su vez el análisis tafonómico indica la intervención de diversos agentes y procesos que supeditan la formación de los conjuntos zooarqueológicos, lo cual 
también ha sido identificado para otros contextos costeros de Patagonia y Tierra del Fuego (Borella y Borrero 2010, 2019; Borella y Muñoz 2006; Borrero 2004; Cruz y Muñoz 2010; Muñoz 1996, 2003, 2008, 2009, 2011, 2014; Muñoz y Cruz 2014; Muñoz et al. 2013; Vázquez et al. 2019). En el análisis presentado en este trabajo, al considerar el potencial de supervivencia de los diferentes elementos óseos, los perfiles anatómicos identificados en el registro arqueológico no son persuasivos sobre las estrategias de transporte y procesamiento de las carcasas y cuestionan la utilidad de los modelos e índices de utilidad económica en otáridos para un estudio con perspectiva arqueológica. Esto se observa a partir de una mayor presencia de elementos de poco valor económico, puesto que muestran cierto solapamiento con aquellos pertenecientes al conjunto de alto potencial de supervivencia, particularmente los huesos largos.

En términos generales, los aspectos relacionados con la preservación de los restos óseos de estos mamíferos marinos han sido menos analizados en relación con mamíferos terrestres, además que la discusión sobre el aprovechamiento de estas presas se ha centrado mayormente en preguntas específicas relacionadas especialmente con las áreas de captura y su relación con la tecnología disponible (Borella 2006; Hildebrandt y Jones 1992; Jones y Hildebrandt 1995; Lyman 1989, 1995; entre otros). No obstante, al incorporar análisis específicos sobre los conjuntos de pinnípedos es posible observar cierta variabilidad previamente poco descripta, de la que podemos a dar cuenta a partir del desarrollo de métodos particulares centrados en el estudio de estas presas.

Agradecimientos: Este trabajo se ha desarrollado en el marco del PICT 1230-2017. A Luis A. Orquera y Ernesto L. Piana por brindarnos acceso a las colecciones de Túnel I y Kaiyawoteha II. Agradecemos a Daniela Alunni, Martín Vázquez y a dos evaluadores anónimos por las lecturas y los valiosos comentarios sobre versiones previas de este manuscrito.

\section{Notas}

1 Para realizar la correlación se consideraron los valores de densidad mineral ósea establecidos en Borella et al. (2007: 424, tabla 1) para huesos enteros, y en el caso de no ser informados, optamos por seleccionar el valor de densidad más bajo, para evitar sobredimensionar el potencial de supervivencia. Dicha estrategia se utilizó a lo largo de todo el trabajo, considerando que los restos óseos de pinnípedos estudiados previamente en la costa sur de Tierra del Fuego tienden a aparecer enteros (Martinoli 2018; Orquera y Piana 1999).

\section{Bibliografía citada}

\section{Alunni, D., A. Tivoli y A. F. Zangrando}

2020 Movilidad y explotación de recursos terrestres por cazadores-recolectores costeros de Bahía Moat, costa sureste de Tierra del Fuego, Argentina. Latin American Antiquity: 1-19. 
Alunni, D. y A. F. Zangrando

2012 Primeros datos sobre el transporte, procesamiento y consumo de guanaco en la localidad arqueológica Heshkaia (sudeste de Tierra del Fuego, Argentina). Magallania 40(1): 319-331.

Alunni, D., A. F. Zangrando y M. A. Gutiérrez

2017 Natural accumulation and distribution of guanaco bones in the southernmost tip of Tierra del Fuego (Argentina): taphonomic analysis and archaeological implications. Journal of Taphonomy 15(1-3): 59-76.

Ames, $\mathrm{K}$.

2002 Going by Boat. The Forager-Collector Continuum at Sea. Beyond Foraging and Collecting. Evolutionary Change in Hunter-Gatherer Settlement System (ed. por B. Fitzhugh y J. Habu, pp. 19-52. Kluwer Academic/Plenum Publishers, Nueva York.

Behrensmeyer, A. K.

1975 Taphonomy and paleoecology in the hominid fossil record. Yearbook of Physical Anthropology 19: 36-50.

1978 Taphonomic and Ecologic Information from Bone Weathering. Paleobiology 5: 150-162.

Belardi, J. B., D. Rindel y T. Bourlot

2012 Much more than it was expected: preservational differences of diaphysis and epiphyseal ends of guanaco (Lama guanicoe) long bones in southern Patagonia (Argentina). Journal of Taphonomy 10(1): 45-65.

Blumenschine, R.

1988 An experimental model of the timing of hominid and carnivore influence of early archaeological bone assemblages. Journal of Archaeological Science 15: 483-501.

Binford, L.

1981 Bones: Ancient Men and Modern Myths. Academic Press, Nueva York.

Borella, F.

2004 Tafonomía regional y estudios arqueofaunísticos de cetáceos en Tierra del Fuego y Patagonia meridional (Vol. 1257). British Archaeological Reports, Oxford.

2006 ¿Dónde están los lobos en la costa atlántica de norpatagonia? Explorando vías para resolver el registro arqueofaunístico. Werken 9: 97-114.

Borella, F. y L. A. Borrero

2010 Observaciones tafonómicas acerca de la desarticulación de carcasas de pinnípedos en ambientes litorales, el caso de Islote Lobos (Golfo San Matías, Río Negro). Zooarqueología a principios del siglo XXI: Aportes teóricos, metodológicos y casos de 
estudio (ed. por M. A. Gutiérrez, M. De Nigris, P. Fernández, M. Giardina, A. Gil, A. Izeta, G. Neme y H. Yacobaccio), pp. 371-379. Ediciones del Espinillo, Buenos Aires.

2019 Exploración de la visibilidad de los restos de otáridos subadultos en acumulaciones óseas naturales recientes próximas a una lobería reproductiva en Nordpatagonia (Río Negro, Argentina). Cuadernos del Instituto Nacional de Antropología y Pensamiento Latinoamericano (Series Especiales) 7 (2): 43-51.

Borella, F., R. Frontini y C. Bayón

2017 Exploring the role of otariids in the subsistence of hunter-gatherers from Pampean archaeological sites at the Late Holocene (Argentina). Journal of Archaeological Science: Reports 12: 142-150.

Borella, F., F. Grandi, D. Vales, N. R. Goodall y E. Crespo

2013 Esquema preliminar de fusión epifisaria en huesos de lobos marinos (Arctocephalus australis y Otaria flavescens), su contribución en los análisis zooarqueológicos. Tendencias teórico-metodológicas y casos de estudio en la arqueología de Patagonia (ed. por A. F. Zangrando, R. Barberena, A. Gil, G. Neme, M. Giardina, L. Luna, C. Otaola, S. Paulides, L. Salgán y A. Tivoli), pp. 39-51. Museo de Historia Natural, San Rafael.

Borella, F., M. A. Gutiérrez, H. Fodere y J. Merlo

2007 Estudio de densidad mineral ósea para dos especies de otáridos frecuentes en el registro arqueofaunístico patagónico (Otaria flavescens y Arctocephalus australis). Arqueología de Fuego-Patagonia. Levantando Piedras, Desenterrando Huesos y Develando Arcanos (ed. por F. Morello, A. Prieto, M. Martinic y G. Bahamonde), pp. 421-426. Ediciones CEQUA, Punta Arenas.

Borella, F. y S. A. Muñoz

2006 Observaciones tafonómicas sobre restos de pinnípedos en la costa norte fueguina (Argentina). Intersecciones en Antropología 7: 399-403.

Borella, F., D. Vales, F. Grandi y N. García

2018 Rasgos diagnósticos en elementos postcraneales de dos especies de otáridos para su identificación en el registro zooarqueológico. Magallania (Punta Arenas) 46(2): 187-203.

Borrero, L. A.

1988 Estudios tafonómicos en Tierra del Fuego: su relevancia para entender procesos de formación del registro arqueológico. Arqueología contemporánea argentina: actualidad y perspectivas: 13-32. Ediciones Búsqueda, Buenos Aires.

1990 Taphonomy of Guanaco Bones in Tierra del Fuego. Quaternary Research 34: 361-371.

2004 Tafonomía Regional: el caso de los pinnípedos. Contra viento y marea. Arqueología de Patagonia (ed. por M. Civalero, P. Fernández y A. Guráieb), pp. 445-454. INAPL, Buenos Aires. 
2007 Longitudinal taphonomic studies in Tierra del Fuego, Argentina. BAR International Series 1601, 219.

Brain, C. K.

1981 The hunters or the hunted? An introduction to African cave taphonomy. Chicago, Chicago University Press.

Carlson, K. y T. Pickering

2004 Shape-adjusted bone mineral density measurements in baboons: other factors explain primate skeletal element representation at Swartkrans. Journal of Archaeological Science 31(5): 577-583.

Corbat, M., A. Tivoli y A. F. Zangrando

2020 Aprovisionamiento de peces en el extremo oriental del canal Beagle (Tierra del Fuego, Argentina): una evaluación a partir del registro zooarqueológico de Bahía Moat. Archaeofauna: International Journal of Archaeozoology 29: 41-57.

Cruz, I.

2003 Paisajes tafonómicos de restos de Aves en el sur de Patagonia continental. Aportes para la interpretación de conjuntos avifaunísticos en registros arqueológicos del Holoceno. Tesis Doctoral inédita, Facultad de Filosofía y Letras, Universidad de Buenos Aires, Buenos Aires.

2007 The recent bones of the Rio Gallegos basin (Santa Cruz, Argentina) and their preservation potential. Taphonomy and Zooarchaeology in Argentina (ed. por M. A. Gutiérrez, L. Miotti, G. Barrientos, G. M. Goñalons, M. Salemme), pp. 161-170.BAR International Series 1601, Oxford.

2015 Las investigaciones sobre preservación de huesos de aves y mamíferos grandes en Patagonia (Argentina). Archaeofauna 24(1): 209-224.

\section{Cruz, I., C. A. Kaufmann, M. Recofsky, M. Álvarez y M. A. Gutiérrez}

2019 Indicadores de edad en huesos de pingüinos patagónicos (Spheniscus magellanicus). Aportes para la discusión sobre la explotación de áreas de nidificación. Cuadernos del Instituto Nacional de Antropología y Pensamiento Latinoamericano 7: 76-87.

Cruz, I. y S. A. Muñoz

2010 Tafonomía comparativa: seguimiento de carcasas de mamíferos en Punta Entrada (Santa Cruz, Argentina). Zooarqueología a Principios Del Siglo XXI: Aportes Teóricos, Metodológicos y Casos de Estudio (ed. por M. Gutiérrez, M. De Nigris, P. Fernández, M. Giardina, A. Gil, A. Izeta, G. Neme, H. Yacobaccio), pp. 387-396. Ediciones del Espinillo, Buenos Aires.

Cruz, I., S. A. Muñoz, B. Ercolano, C. Lemaire, A. Pretto, G. Nauto y C. Moreno 2015 Apostaderos de pinnípedos en Punta Entrada (Santa Cruz, Patagonia Argentina). Explotación Humana e Historia Natural. Magallania 43(1): 291-308. 
Estévez, E. y L. Mameli

2000 Muerte en el Canal: experiencias bioestratinómicas controladas sobre la acción sustractora de Cánidos. Archaeofauna 9: 7-16.

Frontini, R., Y. Fernández-Jalvo, M. Pesquero Fernández, R. Vecchi y C. Bayón 2019 Abrasion in archaeological fish bones from sand dunes. An experimental approach. Archaeological and Anthropological Sciences11: 4891-4907

Gutiérrez, M. A., C. A. Kaufmann, M. E. González, A. Massigoge y M. C. Álvarez 2010 Intrataxonomic variability in metapodial and femur bone density related to age in guanaco (Lama guanicoe). Zooarchaeological and taphonomical implications. Journal of Archaeological Science 37(12): 3226-3238.

Gutiérrez, M. A., C. A. Kaufmann, M. E. González, N. Scheifler, D. Rafuse, A. Massigoge y M. C. Álvarez

2016 The role of small carnivores in the movement of bones: implications for the Pampas archaeofaunal record, Argentina. Archaeological and Anthropological Sciences 8(2): 257-276.

Hildebrandt, W. y T. Jones

1992 Evolution of Marine Mammal Hunting: A view from the California and Oregon Coast. Journal Anthropological Archaeology 11(4): 360-401.

Jones, T. y W. Hildebrandt

1995 Reasserting a Prehistoric Tragedy of the Commons: Reply to Lyman. Journal of Anthropological Archaeology 14(1): 78-98.

Kaufmann, C. A.

2016 Análisis tafonómico de una asociación faunística generada por pequeños carnívoros de la Región. Intersecciones en Antropología 17 (3): 363-373.

Legoupil, D.

1989 Punta Baja. Ethno-Archéologie dans les archipels de Patagonia: les nomades marines de Punta Baja. Editions Recherche sur les Civilisations, París.

Lupo, K.

2006 What explains the carcass field processing and transport decisions of contemporary hunter-gatherers? Measures of economic anatomy and zooarchaeological skeletal part representation. Journal of Archaeological Method and Theory 13(1): 19-66.

Lyman, R. L.

1989 Seal and Sea lion Hunting: A Zooarchaeological Study from the Southern Northwest Coast of North America. Journal of Anthropological Archaeology 8: 68-99. 
1994 Vertebrate Taphonomy. Cambridge University Press, Cambridge.

1995 On the Evolution of Marine Mammal Hunting on the West Coast of North America. Journal of Anthropological Archaeology 14: 45-77.

Lyman, R L., J. Savelle y P. Whitridge

1992 Derivation and application of a meat utility index for phocid seals. Journal of Archaeological Science 19(5): 531-555.

Marean, C. y N. Cleghorn

2003 Large Mammal Skeletal Element Transport: Applying Foraging Theory in a Complex Taphonomic System. Journal of Taphonomy 1 (1): 15-42.

Marean, C., L. Spencer, R. Blumenschine y S. Capaldo

1992 Captive hyaena bone choice and destruction, the schlep effect and Olduvai Archaeofaunas. Journal of Archaeological Science 19: 101-121.

Martín, F.

1998 Madrigueras, dormideros y letrinas: aproximación a la tafonomía de zorros. Arqueología de la Patagonia Meridional (Proyecto Magallania) (ed. por L. Borrero), pp. 7396. Ediciones Búsqueda de Ayllu, Entre Ríos.

Martinoli, M. P.

2017 Pautas de procesamiento y consumo de pinnípedos en la costa sur de Tierra del Fuego e Isla de los Estados: un análisis comparativo. Arqueología 23(3): 173-196.

2018 Modalidades de explotación y procesamiento de pinnípedos en la margen meridional de Tierra del Fuego. Tesis Doctoral inédita, Facultad de Filosofía y Letras, Universidad de Buenos Aires, Buenos Aires.

2019 Anatomía económica del lobo marino de dos pelos (Arctocephalus australis): una porte a la generación de marcos de referencia para especies del neotrópico. Latin American Antiquity 30(3): 587-605.

Martinoli, M. P. y M. Vázquez

2017 Pinniped Capture and Processing: a Comparative Analysis from Beagle Channel (Tierra del Fuego, Argentina). Zooarchaeology in the Neotropics: Environmental Diversity and Human-Animal Interactions (ed. por M. Mondini, S. Muñoz y P. Fernández), pp. 7-23. Springer, Nueva York.

Mondini, M.

2018 Carnivore taphonomy in South America: a review of actualistic studies and their implications in the southern Neotropics. Historical Biology 30(6): 774-785.

Moreno, J.

2008 Arqueología y etnohistoria de la Costa Patagónica Central en el Holoceno Tardío. Fondo Editorial Provincial, Secretaría de Cultura del Chubut, Rawson. 
Muñoz, S. A.

1996 Explotación de pinnípedos en la costa Atlántica de Tierra del Fuego. Arqueología 6: 199-222.

2003 La explotación de mamíferos por cazadores recolectores terrestres de Tierra del Fuego. Tesis Doctoral inédita, Facultad de Filosofía y Letras, Universidad de Buenos Aires, Buenos Aires.

2005 Current perspectives on human-animal relationships in Atlantic Tierra del Fuego island, southern Patagonia. Before Farming 2: 183-196.

2008 Propiedades tafonómicas de las asociaciones óseas de pinnípedos en la costa atlántica de la Isla Grande de Tierra del Fuego, Argentina. Geobios 4: 113-122.

2009 Los cánidos como agentes tafonómicos en los depósitos arqueológicos de la Isla Grande de Tierra del Fuego. Arqueología de Patagonia: una Mirada desde el Último Confín (ed. por M. Salemme, F. Santiago, M. Álvarez, E. Piana, M. Vázquez y E. Mansur), pp. 799-811. Utopías, Ushuaia.

2011 Pinniped Zooarchaeological Studies in Southern Patagonia: Current Issues and Future Research Agenda. Trekking the Shore. Changing Coastlines and the Antiquity of Coastal Settlement (ed. por N. Bicho, J. Haws y L. Davis), pp. 305-331.

2014 La explotación de lobos marinos por cazadores-recolectores terrestres de Tierra del Fuego. Cazadores de mar y tierra. Estudios recientes en arqueología fueguina (ed. por J. Oría y A. Tivoli), pp. 197- 218. Museo del Fin del Mundo, Ushuaia.

Muñoz, S. A. e I. Cruz

2014 Estado actual de las investigaciones tafonómicas naturalistas en Punta Entrada y Monte León (Patagonia Meridional). Revista Chilena de Antropología 29: 102-108.

\section{Muñoz, S. A., I. Cruz, C. Lemaire y A. Pretto}

2013 Los restos arqueológicos de pinnípedos de la desembocadura del río Santa Cruz (Punta Entrada, costa atlántica de Patagonia) en perspectiva regional. Tendencias Teórico Metodológicas y Casos de Estudio en la Arqueología Patagónica, (ed. por A. F. Zangrando; R. Barberena; A. Gil; G. Neme; M. Giardina; L. Luna; C. Otaola; S. Paulides; L. Salgán y A. Tivoli), pp. 459-467. Museo de Historia Natural de San Rafael, Sociedad Argentina de Antropología e Instituto Nacional de Antropología y Pensamiento Latinoamericano, Buenos Aires.

Nagaoka, L., S. Wolverton y B. Fullerton

2008 Taphonomic analysis of the Twilight Beach seals. ANUE Press.

Orquera, L. A. y E. L. Piana

1988 Composición tipológica y datos tecnomorfológicos y tecnofuncionales de los distintos conjuntos arqueológicos del sitio Túnel I (Tierra del Fuego). Relaciones de la Sociedad Argentina de Antropología XVII: 225-235.

1992 Un paso hacia la resolución del palimpsesto. En Análisis espacial en la Arqueología Patagónica (ed. por L. Borrero y J. Lanata). Ayllu, Buenos Aires. 
1999 Arqueología de la región del canal Beagle (Tierra del Fuego, Argentina). Sociedad Argentina de Antropología, Buenos Aires.

2001 Composición de conchales de la costa del canal Beagle (Tierra del Fuego, República Argentina)- Primera Parte. Relaciones de la Sociedad Argentina de Antropología 25: 249-274.

2020 Calibración radiocarbónica en la región del canal Beagle. Relaciones de la Sociedad Argentina de Antropología 45.

Piana, E. L., M. Vázquez y A. Ceraso

2007 Kaiyawoteha. Informe de excavación. Informe preparado a solicitud de LOS PETRELES S.A. para cumplimentar con el Análisis de Impacto al Patrimonio Cultural Provincial, Ley Provincial N³70.

Rasmussen, K.

1931 The Netsilik Eskimos: Social life and spiritual culture (Vol. 8, No. 1-2). Ams Press.

Rindel, D. y J. B. Belardi

2006 Mortandad catastrófica de guanacos por estrés invernal y sus implicaciones arqueológicas: el sitio Alero Los Guanacos 1, lago Cardiel (Provincia de Santa Cruz, Argentina). Magallania (Punta Arenas) 34(1): 139-155.

San Román, M.

2009 Anatomía económica de Otaria flavescens. Zooarqueología en el confín del mundo (ed. por P. López, I. Cartajena, C. García y F. Mena), pp.169-180. Facultad de Estudios del Patrimonio Cultural de la Universidad Internacional SEK-Chile, Santiago de Chile.

Savelle, J., M. Friesen y R. Lyman

1996 Derivation and application of an Otariid Utility Index. Journal of Archaeological Science 23: 705-712.

Schiavini, A.

1990 Estudio de la relación entre el hombre y los pinnípedos en el proceso adaptativo humano del canal Beagle (Tierra del Fuego, Argentina). Tesis Doctoral inédita, Facultad de Ciencias Exactas y Naturales, Universidad de Buenos Aires, Buenos Aires.

1993 Los lobos marinos como recursos para cazadores-recolectores marinos: el caso de Tierra del Fuego. Latin American Antiquity 4: 346-366.

Serrán, M., N. Centeno, N. Weiler y J. Gómez Otero

2008 Massive death of pinnipeds 1200 years ago: taphonomic history of the "Lobos site" (Golfo Nuevo, Patagonia, Argentina). Quaternary International 183(1): 135-142.

Stiner, $\mathrm{M}$.

1991 Food procurement and transport by human and non-human predators. Journal of Archaeological Science 18(4): 455-482. 
2002 On in situ attrition and vertebrate body part profiles. Journal of Archaeological Science 29(9): 979-991.

Stora, J.

2002 Neolithic seal exploitation on the Aland Island in the Baltic sea on the basis of epiphyseal fusion data and metric studies. International Journal of Osteoarchaeology 12: 49-64.

Vaz Ferreira, R. y S. Vallejo

1981 Algunos aspectos del comportamiento de Arctocephalus australis (Zimmerman) lobo de dos pelos de Sudamérica (pinnipedia-otariidae) en el Uruguay. Trabajo presentado en VI Jornadas Argentinas de Zoología, Simposios: 223- 236. La Plata.

Vázquez, M., D. Alunni y A. Valenzuela

2016 Observaciones actualísticas controladas sobre la acción de carroñeros en el Parque Nacional de Tierra del Fuego: implicancias para el registro zooarqueológico. Trabajo presentado en el IV Congreso Nacional de Zooarqueología: 59. UNTDF, Ushuaia.

Vázquez, M., F. Santiago y A. F. Zangrando

2019 La factoría de bahía Thetis, antecedentes históricos y tafonomía de camadas de huesos de pinnípedos. Magallania 47(2): 151-173.

Whitridge, $\mathrm{P}$.

1990 A fauna1 analysis of "warm weather" Thule sites on Somerset Island. Trabajo presentado en Annual Meeting of the Canadian Archaeological Association, Whitehorsc.

Zangrando, A. F.

2010 Coastal archaeology and hunter-gatherers in the south-eastern of Tierra del Fuego. Journal of Island and Coastal Archaeology 5 (2): 288-291.

Zangrando, A. F., D. Alunni, M. P. Martinoli, A. Tivoli y E. L. Piana

2010 Arqueología de la región de Moat (Tierra del Fuego, Argentina): estudios preliminares en la localidad arqueológica Heshkaia. Arqueología Argentina en el Bicentenario de la Revolución de Mayo, Actas XVII Congreso Nacional de Arqueología Argentina: 2005-2010 (ed. por J. Bárcena y H. Chiavazza). Mendoza.

Zangrando, A. F., K. Borrazzo, A. Tivoli, D. Alunni y M. P. Martinoli

2014 El sitio Heshkaia 35: nuevos datos sobre la arqueología de Moat (Tierra del Fuego, Argentina). Revista del Museo de Antropología 7(1): 11-24. 